\title{
Existence for fractional Dirichlet boundary value problem under barrier strip conditions
}

Qilin Song ${ }^{\mathrm{a}}$, Xiaooyu Dong ${ }^{\mathrm{a}}$, Zhanbing Bai ${ }^{\mathrm{a}, *}$, Bo Chen ${ }^{\mathrm{b}}$

${ }^{a}$ College of Mathematics and System Science, Shandong University of Science and Technology, Qingdao 266590, P. R. China.

${ }^{b}$ College of Mathematics and Statistics, Shenzhen University, Shenzhen 518060, P. R. China.

Communicated by X.-J. Yang

\begin{abstract}
In this paper, a fixed-point theorem is used to establish existence results for fractional Dirichlet boundary value problem

$$
D^{\alpha} x(t)=f\left(t, x(t), D^{\alpha-1} x(t)\right), \quad x(0)=A, x(1)=B,
$$

where $1<\alpha \leqslant 2, D^{\alpha} \chi(t)$ is the conformable fractional derivative, and $f:[0,1] \times R^{2} \rightarrow R$ is a continuous function. The main condition is sign condition. The method used is based upon the theory of fixed-point index. (C)2017 All rights reserved.
\end{abstract}

Keywords: Barrier strips, fixed-point index, conformable fractional derivative.

2010 MSC: 34A08, 34B18, 35J05.

\section{Introduction}

Due to the extensive application of fractional calculus in non-Newtonian fluid mechanics, signal analysis, image processing, and other disciplines [2, 3, 35-39], the fractional differential equations have been widely studied and many interesting results have been obtained. For example, in 2005, by using fixed point theorem of cone extension and compression, the existence of positive solution of the following problem are obtained [8]

$$
\left\{\begin{array}{l}
D_{0+}^{\alpha} u(t)+f(t, u(t))=0 \\
u(0)=u(1)=0
\end{array}\right.
$$

After that, the idea was developed to deal with various fractional boundary value problems such as fractional boundary value problem at resonance $[5,6,9,10]$, Caputo fractional derivative problem [41], impulsive problem [7, 31, 33], nonlocal problem [4], integral boundary value problem [27], variational structure problem [21], fractional p-Laplace problem [15, 20, 25, 26, 34, 40], fractional lower and upper

\footnotetext{
${ }^{*}$ Corresponding author

Email addresses: 912078531@qq.com (Qilin Song), dxy17854288324@163.com (Xiaooyu Dong), zhanbingbai@163.com (Zhanbing Bai), chenbo@szu.edu.cn (Bo Chen)

doi:10.22436/jnsa.010.07.19
}

Received 2017-04-14 
solution problem [11, 12, 42], fractional delay problems, [30, 32, 44], solitons [16], Biological mathematics $[17,29,43]$, etc. However, all above works were obtained with standard Riemann-Liouville or Caputo fractional derivatives. The unusual properties of these fractional derivatives lead to some difficulties in application of fractional derivatives in physics and mechanics. Recently, the new conformable fractional derivative definition given by $[1,14,24]$ has many good properties which inspired us study problems with conformable fractional derivative.

In the research of integer order boundary value problem, Kelevedjiev got the existence of the solutions by using the technique of barrier strips in $[22,23]$. These ideas were developed by Ma and Luo [28] and Gao [18] to other problems. Very recently, we obtained the existence of solutions for fractional differential equation

$$
D^{\alpha} x(t)=f\left(t, x(t), D^{\alpha-1} x(t)\right),
$$

with one of the following boundary value conditions

$$
x(0)=A, \quad D^{\alpha-1} x(1)=B, D^{\alpha-1} x(0)=A, \quad x(1)=B,
$$

where $\mathrm{D}^{\alpha} \chi(\mathrm{t})$ is the conformable fractional derivative. The main tool used is the topological transversality theorem [19].

In this paper, by using the fixed-point index theory, the barrier strips technique and a priori estimation, we consider the following problem

$$
D^{\alpha} x(t)=f\left(t, x(t), D^{\alpha-1} x(t)\right), \quad x(0)=A, \quad x(1)=B,
$$

where $1<\alpha \leqslant 2, D^{\alpha} x(t)$ is the conformable fractional order derivative, and $f:[0,1] \times R^{2} \rightarrow R$ is a continuous function. The existence results of solutions to the problem are obtained under $f$ satisfying some sign conditions.

The rest of the paper is organized as follows. In Section 2, the definitions and some preliminaries of the conformable fractional derivative and integral are given. In Section 3, by the use of the fixed-point theorem and the technique of barrier strips, the existence of the solution is obtained. An example is presented to illustrate the main results.

\section{Conformable fractional order calculus}

For the convenience of the reader, we recall some definitions and lemmas which can be found in $[1,13,24]$.

Definition 2.1. Suppose $\alpha \in(n, n+1], u:[0, \infty) \rightarrow R$, and $u$ n-order differentiable for $t>0$, the $\alpha$-order fractional derivative of $u$ is defined as

$$
D^{\alpha} u(t)=\lim _{\varepsilon \rightarrow 0} \frac{u^{(n)}\left(t+\varepsilon t^{n+1-\alpha}\right)-u^{(n)}(t)}{\varepsilon},
$$

provided the limits of the right side exists.

If $u$ is $\alpha$-order differentiable on $(0, a), a>0$, and $\lim _{t \rightarrow 0^{+}} D^{\alpha} u(t)$ exists, then define

$$
\mathrm{D}^{\alpha} \mathfrak{u}(0)=\lim _{t \rightarrow 0^{+}} \mathrm{D}^{\alpha} \mathfrak{u}(\mathrm{t})
$$

Lemma 2.2 ([24]). Let $\alpha \in(n, n+1], u:[0, \infty) \rightarrow R$. Function $u(t)$ is $\alpha$-order differentiable if and only if $u$ is $(\mathrm{n}+1)$-order differentiable, moreover,

$$
D^{\alpha} u(t)=t^{n+1-\alpha} u^{(n+1)}(t)
$$


Definition 2.3. Let $\alpha \in(n, n+1], u:[0, \infty) \rightarrow R$. The $\alpha$-order fractional integral of $u(t)$ is defined as

$$
J_{0+}^{\alpha} u(t)=I^{n+1}\left[t^{\alpha-n-1} u(t)\right]=\frac{1}{n !} \int_{0}^{t}(t-s)^{n} s^{\alpha-n-1} u(s) d s,
$$

where $\mathrm{I}^{\mathrm{n}+1}$ is the $(\mathrm{n}+1)$-order integral.

Remark 2.4. With Lemma 2.2 and Definition 2.3, for $\alpha \in(n, n+1], i=0,1, \cdots, n$, there hold

$$
D^{\alpha-i}\left[J_{0+}^{\alpha} u(t)\right]=t^{n+1-\alpha} D^{n+1-i}\left[I^{n+1}\left(t^{\alpha-n-1} u(t)\right)\right]=t^{n+1-\alpha} I^{i}\left[t^{\alpha-n-1} u(t)\right] .
$$

Lemma 2.5 ([24]). Let $a \geqslant 0, f:[0, b] \rightarrow R$ satisfy

(i) $f$ is continuous on $[0, b]$;

(ii) $f$ is $\alpha$-order differentiable on $(0, b)$,

then, there exists $c \in(a, b)$ such that $D^{\alpha} f(c)=(f(b)-f(a)) /\left(\frac{1}{\alpha} b^{\alpha}-\frac{1}{\alpha} a^{\alpha}\right)$.

Now, we introduce a function space. Given $\alpha \in(n, n+1]$, let

$$
\begin{aligned}
C^{\alpha}[0,1] & =\left\{u \mid u(t)=J_{0^{+}}^{\alpha} x(t)+C_{n} t^{n}+\cdots+C_{1} t+C_{0}, C_{i} \in R, i=0,1 \cdots, n, x(t) \in C[0,1]\right\}, \\
\|u\|_{\alpha} & =\left\|D^{\alpha} u\right\|_{0}+\left\|D^{\alpha-1} u\right\|_{0}+\cdots+\left\|D^{\alpha-n} u\right\|_{0}+\|u\|_{0},
\end{aligned}
$$

where $\|u\|_{0}=\max _{t \in[0,1]}|u(t)|$.

Lemma $2.6([19]) \cdot\left(C^{\alpha}[0,1],\|\cdot\|_{\alpha}\right)$ is a Banach space.

\section{Main results}

Theorem 3.1. Let $f:[0,1] \times R^{2} \rightarrow R$ be continuous. Suppose there are constants $L_{i}, i=1,2, \cdots, 8$ such that $\mathrm{L}_{2}>\mathrm{L}_{1} \geqslant \mathrm{E}, \mathrm{L}_{4}>\mathrm{L}_{3} \geqslant \mathrm{E}, \mathrm{L}_{5}<\mathrm{L}_{6} \leqslant \mathrm{E}, \mathrm{L}_{7}<\mathrm{L}_{8} \leqslant \mathrm{E}$, where $\mathrm{E}=(\alpha-1)(\mathrm{B}-\mathrm{A})$ and

$$
\begin{aligned}
& f(t, x, p) \geqslant 0 \text { for }(t, x, p) \in[0,1] \times R \times\left(\left[L_{1}, L_{2}\right] \cup\left[L_{5}, L_{6}\right]\right), \\
& f(t, x, p) \leqslant 0 \text { for }(t, x, p) \in[0,1] \times R \times\left(\left[L_{3}, L_{4}\right] \cup\left[L_{7}, L_{8}\right]\right) .
\end{aligned}
$$

Then problem (1.1) has at least one solution in $\mathrm{C}^{\alpha}[0,1]$.

Proof. Consider the boundary value problem:

$$
\begin{aligned}
D^{\alpha} x(t) & =\lambda f\left(t, x(t), D^{\alpha-1} x(t)\right), \quad t \in[0,1], \quad \lambda \in[0,1], \\
x(0) & =A, \quad x(1)=B .
\end{aligned}
$$

Define a compact homotopy operator $T_{\lambda}: \mathrm{C}_{\mathrm{B}}^{\alpha}[0,1] \rightarrow \mathrm{C}_{\mathrm{B}}^{\alpha}[0,1]$ as

$$
\left(T_{\lambda} x\right)(t)=\lambda \int_{0}^{1} G(t, s) f\left(s, \chi(s), D^{\alpha-1} \chi(s)\right) d s+\psi(t),
$$

where

$$
C_{B}^{\alpha}[0,1]=\left\{x \in C^{\alpha}[0,1] \mid x(0)=A, x(1)=B\right\}, \quad G(t, s)= \begin{cases}(1-t) s^{\alpha-1}, & 0 \leqslant s \leqslant t \leqslant 1 \\ t s^{\alpha-2}(1-s), & 0 \leqslant t \leqslant s \leqslant 1,\end{cases}
$$

is the Green function (see [13]) and $\psi(t)=A+B t$ is the solution of the problem

$$
\left\{\begin{array}{l}
\mathrm{D}^{\alpha} x(\mathrm{t})=0, \\
x(0)=\mathrm{A}, \quad x(1)=B
\end{array}\right.
$$


Then $T_{\lambda}: C_{B}^{\alpha}[0,1] \rightarrow C_{B}^{\alpha}[0,1]$ is completely continuous (see [13]). The fixed point of $T_{\lambda}$ is the solution of problem (3.1)-(3.2). For some $M>0$, let

$$
\mathrm{U}=\left\{\mathrm{u} \in \mathrm{C}_{\mathrm{B}}^{\alpha}[0,1] \mid\|\mathrm{u}\|_{\alpha}<\mathrm{M}+\|\psi\|_{\alpha}+1\right\} .
$$

We will find positive number $M$ such that $T_{\lambda}: \bar{U} \rightarrow C_{B}^{\alpha}[0,1]$ has no fixed-point on $\partial \mathrm{U}$ for every $\lambda \in[0,1]$.

In fact, all the possible solutions of problem (3.1)-(3.2) have a priori bound that do not depend on $\lambda \in[0,1]$. Let $x(t) \in C^{\alpha}[0,1]$ be a solution to problem (3.1)-(3.2). Firstly, we will estimate $D^{\alpha-1} x(t)$. By Lemma 2.5, there is $d \in(0,1)$ such that $D^{\alpha-1} \chi(d)=(\alpha-1)(B-A)=E$. Then

$$
\begin{aligned}
& f(t, x, p) \geqslant 0 \text { for }(t, x, p) \in[0, d] \times R \times\left[L_{1}, L_{2}\right], \\
& f(t, x, p) \leqslant 0 \text { for }(t, x, p) \in[0, d] \times R \times\left[L_{7}, L_{8}\right] .
\end{aligned}
$$

Assume that the sets

$$
\mathrm{S}_{0}=\left\{\mathrm{t} \in[0, \mathrm{~d}] \mid \mathrm{L}_{1}<\mathrm{D}^{\alpha-1} \chi(\mathrm{t}) \leqslant \mathrm{L}_{2}\right\}, \quad \mathrm{S}_{1}=\left\{\mathrm{t} \in[0, \mathrm{~d}] \mid \mathrm{L}_{3}<\mathrm{D}^{\alpha-1} \chi(\mathrm{t})<\mathrm{L}_{4}\right\},
$$

are not empty. Let $t_{0} \in S_{0}, t_{1} \in S_{1}$ be fixed. Assume there are $t_{0}^{\prime} \in\left(t_{0}, d\right]$ and $t_{1}^{\prime} \in\left(t_{1}, d\right]$ such that

$$
\mathrm{D}^{\alpha-1} x\left(\mathrm{t}_{0}^{\prime}\right)<\mathrm{D}^{\alpha-1} x\left(\mathrm{t}_{0}\right), \quad \mathrm{D}^{\alpha-1} x\left(\mathrm{t}_{1}^{\prime}\right)>\mathrm{D}^{\alpha-1} x\left(\mathrm{t}_{1}\right) .
$$

The continuity of $D^{\alpha-1} \chi(t)$ allows us to take $t_{0}^{\prime} \in\left(t_{0}, d\right] \cap S_{0}, t_{1}^{\prime} \in\left(t_{1}, d\right] \cap S_{1}$. But $D^{\alpha} x(t)=\lambda f(t, x(t)$, $\left.D^{\alpha-1} x(t)\right) \geqslant 0$, for $t \in S_{0}$ and $D^{\alpha} x(t) \leqslant 0$, for $t \in S_{1}$. Consequently,

$$
\mathrm{D}^{\alpha-1} x\left(\mathrm{t}_{0}^{\prime}\right) \geqslant \mathrm{D}^{\alpha-1} x\left(\mathrm{t}_{0}\right), \quad \mathrm{D}^{\alpha-1} x\left(\mathrm{t}_{1}^{\prime}\right) \leqslant \mathrm{D}^{\alpha-1} x\left(\mathrm{t}_{1}\right) .
$$

From the contradiction to (3.3), it follows that

$$
\begin{aligned}
& D^{\alpha-1} x(t) \geqslant D^{\alpha-1} x\left(t_{0}\right), \text { for } t \in\left(t_{0}, d\right], \\
& D^{\alpha-1} x(t) \leqslant D^{\alpha-1} x\left(t_{0}\right), \text { for } t \in\left(t_{1}, d\right],
\end{aligned}
$$

and in particular,

$$
E=D^{\alpha-1} \chi(d) \geqslant D^{\alpha-1} \chi\left(t_{0}\right)>L_{1} \geqslant E, \quad E=D^{\alpha-1} \chi(d) \leqslant D^{\alpha-1} \chi\left(t_{1}\right)<L_{8} \leqslant E .
$$

The contradictions obtained show that $S_{0}, S_{1}$ are empty. Since $D^{\alpha-1} \chi(t) \in C[0,1], L_{4} \leqslant D^{\alpha-1} \chi(t) \leqslant L_{1}$ for $\mathrm{t} \in[0, \mathrm{~d}]$, so

$$
\left|\mathrm{D}^{\alpha-1} \chi(\mathrm{t})\right| \leqslant \max \left\{\left|\mathrm{L}_{1}\right|,\left|\mathrm{L}_{8}\right|\right\}, \quad \mathrm{t} \in[0, \mathrm{~d}] .
$$

Similarly, the facts

$$
\begin{aligned}
& f(t, x, p) \leqslant 0 \text { for }(t, x, p) \in[d, 1] \times R \times\left[L_{3}, L_{4}\right] \\
& f(t, x, p) \geqslant 0 \text { for }(t, x, p) \in[d, 1] \times R \times\left[L_{5}, L_{6}\right]
\end{aligned}
$$

yield that

$$
\left|\mathrm{D}^{\alpha-1} \chi(\mathrm{t})\right| \leqslant \max \left\{\left|\mathrm{L}_{3}\right|,\left|\mathrm{L}_{6}\right|\right\}, \quad \mathrm{t} \in[\mathrm{d}, 1]
$$

Consequently,

$$
\left|\mathrm{D}^{\alpha-1} x(\mathrm{t})\right| \leqslant \max \left\{\left|\mathrm{L}_{1}\right|,\left|\mathrm{L}_{3}\right|,\left|\mathrm{L}_{6}\right|,\left|\mathrm{L}_{8}\right|\right\} \triangleq \mathrm{G}_{1}, \quad \mathrm{t} \in[0,1] .
$$

On the other hand, by Lemma 2.5 , for each $t \in(0,1]$, there exists $c \in(0, t)$ such that

$$
x(t)-x(0)=D^{\alpha-1} x(c) \cdot \frac{1}{\alpha-1} t^{\alpha-1} .
$$

From which we get $|x(t)| \leqslant G_{2}, G_{2}=\max \left\{|A|+G_{1} /(\alpha-1)\right\}$. Finally the differential equation (3.1) together with the continuity of $f$ and a priori estimations of $x(t)$ and $D^{\alpha-1} \chi(t)$ show that $\left|D^{\alpha} x(t)\right| \leqslant G_{2}$ for the constant $G_{2}$ independent of $\lambda$. 
From the above arguments, let $M=G_{1}+G_{2}+G_{3}$, we know

$$
\|x\|_{\alpha}=\left\|D^{\alpha} x\right\|_{0}+\left\|D^{\alpha-1} x\right\|_{0}+\|x\|_{0}<M<\infty .
$$

The above results indicate that $T_{\lambda}$ has no fixed point on $\partial U$ and $T_{\lambda}:[0,1] \times \bar{U} \rightarrow C^{\alpha}[0,1]$ is completely continuous. Denote by $X=\mathrm{C}_{\mathrm{B}}^{\alpha}[0,1]$. With the normality of the fixed-point index, the index of the constant operator $T_{0}(x) \equiv \psi(t)$ on $U$ with respect to $C_{B}^{\alpha}[0,1], i\left(T_{0}, U, X\right)=1$. It follows from the homotopy invariant property of the fixed-point index that

$$
i\left(T_{1}, U, X\right)=i\left(T_{\lambda}, U, X\right)=i\left(T_{0}, U, X\right)=1 .
$$

With the solvability of fixed-point index, $T_{1}$ has a fixed-point in $U$, and so problem (1.1) has a solution in $\overline{\mathrm{u}}$.

The conditions imposed on $f(t, x, p)$ above are local with respect to $t$ and $p$. In the next theorem they are also localized with respect to $x$.

Theorem 3.2. Let $f:[0,1] \times R^{2} \rightarrow R$ be continuous. Suppose there are constants $L_{i}, i=1,2, \cdots, 8$ such that $\mathrm{L}_{2}>\mathrm{L}_{1} \geqslant \mathrm{E}, \mathrm{L}_{4}>\mathrm{L}_{3} \geqslant \mathrm{E}, \mathrm{L}_{5}<\mathrm{L}_{6} \leqslant \mathrm{E}, \mathrm{L}_{7}<\mathrm{L}_{8} \leqslant \mathrm{E}$, where $\mathrm{E}=(\alpha-1)(\mathrm{B}-\mathrm{A})$ and

(1) there is $M \geqslant \max \{|A|,|B|\}$ such that $x f(t, x, 0)>0$ for $|x|>M$;

(2) $f(t, x, p) \geqslant 0$ for $(t, x, p) \in[0,1] \times[-M, M] \times\left(\left[L_{1}, L_{2}\right] \cup\left[L_{5}, L_{6}\right]\right), f(t, x, p) \leqslant 0$ for $(t, x, p) \in[0,1] \times$ $[-\mathrm{M}, \mathrm{M}] \times\left(\left[\mathrm{L}_{3}, \mathrm{~L}_{4}\right] \cup\left[\mathrm{L}_{7}, \mathrm{~L}_{8}\right]\right)$.

Then the problem (1.1) has at least one solution in $\mathrm{C}^{\alpha}[0,1]$.

Proof. If $x \in C^{\alpha}[0,1]$ is a solution of problem (1.1), then $|x(t)| \leqslant M$. In fact, if there is $t_{0} \in(0,1)$ such that $x\left(t_{0}\right)=\max \{x(t) \mid t \in(0,1)\}>M$, and then by Lemma $2.2, u$ is $\alpha$-order differentiable if and only if $u$ is second-order differentiable and

$$
D^{\alpha} u(t)=t^{2-\alpha} u^{\prime \prime}(t), \quad D^{\alpha-1} u(t)=t^{2-\alpha} u^{\prime}(t) .
$$

Assuming that $u(t)$ achieves its maximum at $t_{0} \in(0,1)$, then

$$
u^{\prime \prime}\left(t_{0}\right) \leqslant 0, \quad u^{\prime}\left(t_{0}\right)=0,
$$

consequently

$$
D^{\alpha} x\left(t_{0}\right) \leqslant 0, \quad D^{\alpha-1} x\left(t_{0}\right)=0 .
$$

But according to the condition (1), there is

$$
x\left(t_{0}\right) D^{\alpha} x\left(t_{0}\right)=x\left(t_{0}\right) \lambda f\left(t_{0}, x\left(t_{0}\right), 0\right)>0 .
$$

Thus $D^{\alpha} x\left(t_{0}\right)>0$, a contradiction. So $x(t) \leqslant M$. Proving by the same methods we can get $x(t) \geqslant-M$. In conclusion $|x(t)| \leqslant M$. Furthermore, the proof is not essentially different from the proof of Theorem 3.1 .

Example 3.3. Consider the following boundary value problem:

$$
D^{\frac{3}{2}} x(t)=x(t)-\frac{5}{2} D^{\frac{1}{2}} x(t)+\left[D^{\frac{1}{2}} x(t)\right]^{2}-1, \quad x(0)=0, \quad x(1)=1 .
$$

Choose $\mathrm{E}=0.5, \mathrm{M}=1, \mathrm{~L}_{1}=4, \mathrm{~L}_{2}=5, \mathrm{~L}_{3}=1, \mathrm{~L}_{4}=2, \mathrm{~L}_{5}=-2, \mathrm{~L}_{6}=-1, \mathrm{~L}_{7}=0, \mathrm{~L}_{8}=0.5$, then,

$$
\begin{aligned}
& f(t, x, p) \geqslant 0 \text { for }(t, x, p) \in[0,1] \times[-1,1] \times([4,5] \cup[-2,-1]), \\
& f(t, x, p) \leqslant 0 \text { for }(t, x, p) \in[0,1] \times[-1,1] \times([1,2] \cup[0,0.5]) .
\end{aligned}
$$

By Theorem 3.2, problem (3.4) has at least one solution in $C^{\frac{3}{2}}[0,1]$. 


\section{References}

[1] T. Abdeljawad, On conformable fractional calculus, J. Comput. Appl. Math., 279 (2015), 57-66. 1, 2

[2] R. Almeida, A. B. Malinowska, T. Odzijewicz, Fractional differential equations with dependence on the CaputoKatugampola derivative, J. Comput. Nonlinear Dynam., 11 (2016), 061017-061027. 1

[3] A. Alsaedi, D. Baleanu, S. Etemad, S. Rezapour, On coupled systems of time-fractional differential problems by using a new fractional derivative, J. Funct. Spaces, 2016 (2016), 8 pages. 1

[4] Z.-B. Bai, On positive solutions of a nonlocal fractional boundary value problem, Nonlinear Anal., 72 (2010), 916-942. 1

[5] Z.-B. Bai, On solutions of some fractional m-point boundary value problems at resonance, Electron. J. Qual. Theory Differ. Equ., 2010 (2010), 15 pages. 1

[6] Z.-B. Bai, Solvability for a class of fractional m-point boundary value problem at resonance, Comput. Math. Appl., 62 (2011), 1292-1302. 1

[7] Z.-B. Bai, X.-Y. Dong, C. Yin, Existence results for impulsive nonlinear fractional differential equation with mixed boundary conditions, Bound. Value Probl., 2016 (2016), 11 pages. 1

[8] Z.-B. Bai, H.-S. Lü, Positive solutions for boundary value problem of nonlinear fractional differential equation, J. Math. Anal. Appl., 311 (2005), 495-505. 1

[9] Z.-B. Bai, Y.-H. Zhang, The existence of solutions for a fractional multi-point boundary value problem, Comput. Math. Appl., 60 (2010), 2364-2372. 1

[10] Z.-B. Bai, Y.-H. Zhang, Solvability of fractional three-point boundary value problems with nonlinear growth, Appl. Math. Comput., 218 (2011), 1719-1725. 1

[11] Z.-B. Bai, S. Zhang, S.-J. Sun, C. Yin, Monotone iterative method for fractional differential equations, Electron. J. Differential Equations, 2016 (2016), 8 pages. 1

[12] Y.-J. Cui, Uniqueness of solution for boundary value problems for fractional differential equations, Appl. Math. Lett., 51 (2016), 48-54. 1

[13] X.-Y. Dong, Z.-B. Bai, S.-J. Sun, Positive solutions for some boundary value problems with conformable fractional differential derivatives, (Chinese) Acta Math. Sci. Ser. A Chin. Ed., 37 (2017), 82-91. 2, 3

[14] X.-Y. Dong, Z.-B. Bai, W. Zhang, Positive solutions for nonlinear eigenvalue problems with conformable fractional differential derivatives, J. Shandong Univ. Sci. Technol. Nat. Sci., 35 (2016), 85-90. 1

[15] X.-Y. Dong, Z.-B. Bai, S.-Q. Zhang, Positive solutions to boundary value problems of p-Laplacian with fractional derivative, Bound. Value Probl., 2017 (2017), 15 pages. 1

[16] H. H. Dong, B. Y. Guo, B. S. Yin, Generalized fractional supertrace identity for Hamiltonian structure of NLSMKdV hierarchy with self-consistent sources, Anal. Math. Phys., 6 (2016), 199-209. 1

[17] T. Feng, X.-Z. Meng, L.-D. Liu, S.-J. Gao, Application of inequalities technique to dynamics analysis of a stochastic eco-epidemiology model, J. Inequal. Appl., 2016 (2016), 29 pages. 1

[18] C.-H. Gao, Existence of solutions to p-Laplacian difference equations under barrier strips conditions, Electron. J. Differential Equations, 2007 (2007), 6 pages. 1

[19] L.-M. He, X.-Y. Dong, Z.-B. Bai, B. Chen, Solvability of some two-point fractional boundary value problems under barrier strip conditions, (to appear). 1, 2.6

[20] Y.-X. Hua, X.-H. Yu, On the ground state solution for a critical fractional Laplacian equation, Nonlinear Anal., 87 (2013), $116-125.1$

[21] F. Jiao, Y. Zhou, Existence results for fractional boundary value problem via critical point theory, Internat. J. Bifur. Chaos Appl. Sci. Engrg., 22 (2012), 17 pages. 1

[22] P. Kelevedjiev, Existence of solutions for two-point boundary value problems, Nonlinear Anal., 22 (1994), 217-224. 1

[23] P. S. Kelevedjiev, S. Tersian, Singular and nonsingular first-order initial value problems, J. Math. Anal. Appl., 366 (2010), 516-524. 1

[24] R. Khalil, M. Al Horani, A. Yousef, M. Sababheh, A new definition of fractional derivative, J. Comput. Appl. Math., 264 (2014), 65-70. 1, 2, 2.2, 2.5

[25] X.-P. Liu, M. Jia, W.-G. Ge, Multiple solutions of a p-Laplacian model involving a fractional derivative, Adv. Difference Equ., 2013 (2013), 12 pages. 1

[26] X.-P. Liu, M. Jia, W.-G. Ge, The method of lower and upper solutions for mixed fractional four-point boundary value problem with p-Laplacian operator, Appl. Math. Lett., 65 (2017), 56-62. 1

[27] X.-P. Liu, M. Jia, B.-F. Wu, Existence and uniqueness of solution for fractional differential equations with integral boundary conditions, Electron. J. Qual. Theory Differ. Equ., 2009 (2009), 10 pages. 1

[28] R.-Y. Ma, H. Luo, Existence of solutions for a two-point boundary value problem on time scales, Appl. Math. Comput., 150 (2004), 139-147. 1

[29] X.-Z. Meng, S.-N. Zhao, T. Feng, T.-H. Zhang, Dynamics of a novel nonlinear stochastic SIS epidemic model with double epidemic hypothesis, J. Math. Anal. Appl., 433 (2016), 227-242. 1

[30] Z. Wang, A numerical method for delayed fractional-order differential equations, J. Appl. Math., 2013 (2013), 7 pages. 1

[31] G.-T. Wang, B. Ahmad, L.-H. Zhang, J. J. Nieto, Comments on the concept of existence of solution for impulsive fractional differential equations, Commun. Nonlinear Sci. Numer. Simul., 19 (2014), 401-403. 1

[32] Z. Wang, X. Huang, J.-P. Zhou, A numerical method for delayed fractional-order differential equations: based on G-L definition, Appl. Math. Inf. Sci., 7 (2013), 525-529. 1 
[33] J.-R. Wang, Y. Zhou, M. Fečkan, On recent developments in the theory of boundary value problems for impulsive fractional differential equations, Comput. Math. Appl., 64 (2012), 3008-3020. 1

[34] S.-S. Yan, J.-F. Yang, X.-H. Yu, Equations involving fractional Laplacian operator: compactness and application, J. Funct. Anal., 269 (2015), 47-79. 1

[35] X.-J. Yang, A new fractional derivative without singular kernel: Application to the modelling of the steady heat flow, Therm. Sci., 20 (2016), 753-756. 1

[36] X.-J. Yang, Fractional Maxwell fluid with fractional derivative without singular kernel, Therm. Sci., 20 (2016), S871-S877.

[37] X.-J. Yang, Fractional derivatives of constant and variable orders applied to anomalous relaxation models in heat-transfer problems, Therm. Sci., 3 (2017), 1161-1171.

[38] X.-J. Yang, D. Baleanu, H. M. Srivastava, Local fractional integral transforms and their applications, Elsevier/Academic Press, Amsterdam, (2015).

[39] X.-J. Yang, J. A. T. Machado, A new fractional operator of variable order: Application in the description of anomalous diffusion, Phys. A, 481 (2017), 276-283. 1

[40] X.-H. Yu, Solutions of fractional Laplacian equations and their Morse indices, J. Differential Equations, 260 (2016), 860-871. 1

[41] S.-Q. Zhang, Positive solutions for boundary-value problems of nonlinear fractional differential equations, Electron. J. Differential Equations, 2006 (2006), 12 pages. 1

[42] W. Zhang, Z.-B. Bai, S.-J. Sun, Extremal solutions for some periodic fractional differential equations, Adv. Difference Equ., 2016 (2016), 8 pages. 1

[43] T.-Q. Zhang, X.-Z. Meng, Y. Song, T.-H. Zhang, A stage-structured predator-prey SI model with disease in the prey and impulsive effects, Math. Model. Anal., 18 (2013), 505-528. 1

[44] Y.-M. Zou, Y.-J. Cui, Existence results for a functional boundary value problem of fractional differential equations, Adv. Difference Equ., 2013 (2013), 25 pages. 1 